Pacific Journal of Mathematics

THE SPACE OF INFINITE-DIMENSIONAL COMPACT AND
OTHER TOPOLOGICAL COPIES OF $\left(l_{f}^{2}\right)^{\omega}$ ( 


\title{
THE SPACE OF INFINITE-DIMENSIONAL COMPACTA AND OTHER TOPOLOGICAL COPIES OF $\left(l_{f}^{2}\right)^{\omega}$
}

\author{
J. J. Dijkstra, J. VAN Mill, AND J. Mogilski
}

To Doug Curtis, on the occasion of his retirement

We show that there exists a homeomorphism from the hyperspace of the Hilbert cube $Q$ onto the countable product of Hilbert cubes such that the $\geq k$-dimensional sets are mapped onto $B^{k} \times Q \times Q \times$ $\cdots$, where $B$ is the pseudoboundary of $Q$. In particular, the infinitedimensional compacta are mapped onto $B^{\omega}$, which is homeomorphic to the countably infinite product of $l_{f}^{2}$. In addition, we prove for $k \in\{1,2, \ldots, \infty\}$ that the space of uniformly $\geq k$-dimensional sets in $2^{Q}$ is also homeomorphic to $\left(l_{f}^{2}\right)^{\omega}$.

1. Introduction. If $X$ is a compact metric space then $2^{X}$ denotes the hyperspace of $X$ equipped with the Hausdorff metric. According to Curtis and Schori [6] $2^{X}$ is homeomorphic to the Hilbert cube $Q$ whenever $X$ is a nontrivial Peano continuum.

Our primary interest is the subset of $2^{Q}$ consisting of all infinitedimensional compacta. This space is an $F_{\sigma \delta}$-set in $2^{Q}$ and one may expect that it is homeomorphic to the countable product of the preHilbert space

$$
l_{f}^{2}=\left\{x \in l^{2}: x_{i}=0 \text { for all but finitely many } i\right\} .
$$

We prove this conjecture. The space $\left(l_{f}^{2}\right)^{\omega}$ is in a sense maximal in the class $\mathscr{F}_{\sigma \delta}$ of absolute $F_{\sigma \delta}$-spaces and it has received a lot of attention in recent years because of its topological equivalence to numerous function spaces, see e.g. Dijkstra et al. [7].

For $k \in\{0,1,2, \ldots, \infty\}$ we let $\operatorname{Dim}_{\geq k}(X)$ denote the subspace consisting of all $\geq k$-dimensional elements of $2^{X}$. We define $\operatorname{Dim}_{k}(X)$ and $\operatorname{Dim}_{\leq k}(X)$ in the same way. Let $\overline{\operatorname{Dim}}_{\geq k}(X)$ stand for all uniformly $\geq \bar{k}$-dimensional compacta in $2^{X}$, i.e. spaces such that every nonempty open subset is at least $k$-dimensional. The default value here is $X=Q$, i.e., $\operatorname{Dim}_{\geq k}=\operatorname{Dim}_{\geq k}(Q)$ etc.

Let $I$ stand for the interval $[0,1]$. The Hilbert cube is denoted by $Q=\prod_{i=1}^{\infty} I$ with metric $d(x, y)=\max \left\{2^{-i}\left|x_{i}-y_{i}\right|: i \in \mathbf{N}\right\}$. The pseudointerior of $Q$ is $s=\prod_{i=1}^{\infty}(0,1)$ and $B=Q \backslash s$ is the pseudoboundary. 
THEOREM 1.1. (a) There exists a homeomorphism $\alpha$ from $2^{Q}$ onto $Q^{\mathbf{N}}=\prod_{i=1}^{\infty} Q$ such that for every $k \in\{0,1,2, \ldots\}$,

$$
\alpha\left(\operatorname{Dim}_{\geq k}\right)=\underbrace{B \times \cdots \times B}_{k \text { times }} \times Q \times Q \times \cdots .
$$

This implies that $\alpha\left(\operatorname{Dim}_{\infty}\right)=B^{\mathbf{N}}$.

(b) There exists a homeomorphism $\beta$ from $2^{Q}$ onto $Q^{\mathbf{N}}$ such that for every $k \in\{0,1,2, \ldots\}$,

$$
\beta\left(\operatorname{Dim}_{\leq k}\right)=\underbrace{Q \times \cdots \times Q}_{k \text { times }} \times s \times s \times \cdots .
$$

The pseudoboundary $B$ is an absorber for the collection of $\sigma$ compacta $\mathscr{F}_{\sigma}$. Furthermore, $B^{\mathbf{N}}$ is an absorber in $Q^{\mathbf{N}}$ for the collection $\mathscr{F}_{\sigma \delta}$. For definitions see $\S 2$ and $\S 3$. The space $B^{\mathbf{N}}$ is homeomorphic to $\left(l_{f}^{2}\right)^{\omega}$. If $Y$ is an $\mathscr{F}_{\sigma \delta}$-absorber in $Q$, i.e., the pair $(Q, Y)$ is homeomorphic to $\left(Q^{\mathbf{N}}, B^{\mathbf{N}}\right)$, then we have the following:

THEOREM 1.2. There exists a homeomorphism $\alpha$ from $2 Q$ onto $Q^{\mathbf{N}}$ such that for every $k \in\{0,1,2, \ldots\}$,

$$
\alpha\left(\overline{\operatorname{Dim}}_{\geq k}\right)=\underbrace{Y \times \cdots \times Y}_{k \text { times }} \times Q \times Q \times \cdots .
$$

This means that $\overline{\operatorname{Dim}}_{\geq k}$ is homeomorphic to $B^{\mathbf{N}}$ and $\left(l_{f}^{2}\right)^{\omega}$ for $k \in$ $\{1,2, \ldots, \infty\}$.

In the final section we illustrate the power of the technique that we developed to prove the main theorems by applying the method to function spaces $C_{p}(X)$.

For an explanation of undefined terminology see van Mill [12].

2. Absorbing systems. Let $\Gamma$ be an ordered set and let $\mathscr{M}_{\gamma}$ be a collection of spaces for each $\gamma \in \Gamma$. Each $\mathscr{M}_{\gamma}$ is assumed to be topological and closed hereditary. Let $\mathscr{M}$ stand for the whole system $\left(\mathscr{M}_{\gamma}\right)_{\gamma \in \Gamma}$. Let $X=\left(X_{\gamma}\right)_{\gamma \in \Gamma}$ be an order preserving indexed collection of subsets of a topological copy $E$ of $Q$, i.e., $X_{\gamma} \subset X_{\gamma^{\prime}}$ if and only if $\gamma \leq \gamma^{\prime}$.

The system $X$ is called $\mathscr{M}$-universal if for every order preserving system $\left(A_{\gamma}\right)_{\gamma}$ in $Q$ such that $A_{\gamma} \in \mathscr{M}_{\gamma}$ for every $\gamma \in \Gamma$, there is an embedding $f: Q \rightarrow E$ with $f^{-1}\left(X_{\gamma}\right)=A_{\gamma}$. The system $X$ is called strongly $\mathscr{M}$-universal if for every order preserving system $\left(A_{\gamma}\right)_{\gamma}$ in $Q$ such that $A_{\gamma} \in \mathscr{M}_{\gamma}$ for every $\gamma \in \Gamma$, and for every map $f: Q \rightarrow E$ 
that restricts to a Z-embedding on some compact set $K$, there exists a Z-embedding $g: Q \rightarrow E$ that can be chosen arbitrarily close to $f$ with the properties: $g|K=f| K$ and $g^{-1}\left(X_{\gamma}\right) \backslash K=A_{\gamma} \backslash K$ for every $\gamma$. The system $X$ is called reflexively universal if for every map $f: E \rightarrow E$ that restricts to a Z-embedding on some compact set $K$, there exists a Z-embedding $g: E \rightarrow E$ that can be chosen arbitrarily close to $f$ with the properties: $g|K=f| K$ and $g^{-1}\left(X_{\gamma}\right) \backslash K=X_{\gamma} \backslash K$ for every $\gamma$. Observe that $X$ is strongly $\mathscr{M}$-universal whenever $X$ is $\mathscr{M}$-universal and reflexively universal. If $X_{\gamma} \in \mathscr{M}_{\gamma}$ then the converse is also true.

The system $X$ is called $\mathscr{M}$-absorbing if

(1) $X_{\gamma} \in \mathscr{M}_{\gamma}$ for every $\gamma \in \Gamma$,

(2) $\bigcup\left\{X_{\gamma}: \gamma \in \Gamma\right\}$ is contained in a $\sigma$ Z-set of $E$, and

(3) $X$ is strongly $\mathscr{M}$-universal.

This notion appears to be a successful synthesis of the $Q$-matrices technique of van Mill [11] and the generalized absorbers of Bestvina and Mogilski [2]. The power of the method we introduce here comes mainly from the relative ease of application.

As expected we have a uniqueness theorem for absorbing systems:

THEOREM 2.1. If $X$ and $Y$ are both $\mathscr{M}$-absorbing systems in $E$ respectively $E^{\prime}$ then $(E, X)$ and $\left(E^{\prime}, Y\right)$ are homeomorphic, i.e., there is a homeomorphism $h: E \rightarrow E^{\prime}$ such that $h\left(X_{\gamma}\right)=Y_{\gamma}$ for all $\gamma \in \Gamma$. If $E=E^{\prime}$ then the map $h$ can be found arbitrarily close to the identity.

Proof. This is a standard back and forth argument. Obviously, we may assume that $E=E^{\prime}=Q$. Let $\bigcup_{\gamma} X_{\gamma} \subset \bigcup_{i} A_{i}$ and let $\bigcup_{\gamma} Y_{\gamma} \subset$ $\bigcup_{i} B_{i}$, where $\varnothing=A_{0} \subset A_{1} \subset A_{2} \subset \cdots$ and $\varnothing=B_{0} \subset B_{1} \subset B_{2} \subset$ ... are sequences of Z-sets in $Q$. By induction we shall construct sequences of homeomorphisms $f_{i}: Q \rightarrow Q$ and $g_{i}=f_{i} \circ \cdots \circ f_{0}$ with the properties:

$$
\begin{gathered}
A_{i} \cap X_{\gamma}=A_{i} \cap g_{i}^{-1}\left(Y_{\gamma}\right), \quad B_{i} \cap g_{i}\left(X_{\gamma}\right)=B_{i} \cap Y_{\gamma}, \\
f_{i} \mid\left(g_{i-1}\left(A_{i-1}\right) \cup B_{i-1}\right)=1,
\end{gathered}
$$

where 1 denotes the identity map. Put $f_{0}=1$.

Assume that $f_{i}$ has been constructed. Since $X_{\gamma} \in \mathscr{M}_{\gamma}$ and $\mathscr{M}_{\gamma}$ is topological and closed hereditary we have $g_{i}\left(X_{\gamma}\right) \cap\left(g_{i}\left(A_{i+1}\right) \cup B_{i}\right) \in$ $\mathscr{M}_{\gamma}$. Put $K=g_{i}\left(A_{i}\right) \cup B_{i}$ and observe that $g_{i}\left(X_{\gamma}\right) \cap K=Y_{\gamma} \cap K$. Since $Y$ is strongly universal we can find a Z-embedding $\alpha: g_{i}\left(A_{i+1}\right) \cup B_{i} \rightarrow$ $Q$ that fixes $K$ and that has the property

$$
\alpha^{-1}\left(Y_{\gamma}\right) \cap g_{i}\left(A_{i+1}\right)=g_{i}\left(X_{\gamma} \cap A_{i+1}\right) \text {. }
$$


Let $\tilde{\alpha}$ be an extension of $\alpha$ to a homeomorphism of $Q$. Since $\tilde{\alpha} \circ g_{i}(X)$ is just as $X$ strongly universal we can find a Z-embedding $\beta: \alpha \circ g_{i}\left(A_{i+1}\right) \cup B_{i+1} \rightarrow Q$ that fixes $K^{\prime}=\alpha \circ g_{i}\left(A_{i+1}\right) \cup B_{i}$ and that has the property

$$
\beta^{-1}\left(\tilde{\alpha} \circ g_{i}\left(X_{\gamma}\right)\right) \cap B_{i+1}=Y_{\gamma} \cap B_{i+1} .
$$

Let $\tilde{\beta}$ be an extension of $\beta$ to a homeomorphism of $Q$. If we put $f_{i+1}=\tilde{\beta}^{-1} \circ \tilde{\alpha}$ then one can easily verify the induction hypothesis for $i+1$. Since $\tilde{\alpha}$ and $\tilde{\beta}$ and hence $f_{i+1}$ can be chosen arbitrarily close to the identity we may assume that $h=\lim _{i \rightarrow \infty} g_{i}$ is a homeomorphism of $Q$. The function $h$ maps each $X_{\gamma}$ onto $Y_{\gamma}$.

3. Absorbing sequences in $Q^{\mathrm{N}}$. We shall now consider the special case that the system $X$ is a decreasing sequence $Q \supset X_{1} \supset X_{2} \supset$ ... Formally, this corresponds to choosing $\Gamma=\mathbf{N}$ with an inverted ordering. As a further simplification we assume that all the $\mathscr{M}_{y}$ 's are equal to a fixed $\mathscr{M}$ and use the term $\mathscr{M}$-absorbing sequence. In addition, if $\Gamma$ is a singleton then we call $X$ an $\mathscr{M}$-absorber. Recall that the pseudoboundary $B$ of $Q$ is an $\mathscr{F}_{\sigma}$-absorber, where $\mathscr{F}_{\sigma}$ is the collection of $\sigma$-compact spaces. Observe that if $X$ is an $\mathscr{M}$ absorbing sequence and $\mathscr{M}$ is closed under finite intersections then $X_{\infty}=\bigcap_{i=1}^{\infty} X_{i}$ is an $\mathscr{M}_{\delta}$-absorber, where $\mathscr{M}_{\delta}$ stands for the collection of countable intersections of elements of $\mathscr{M}$.

Let $X$ be a subset of $Q$. We define three decreasing sequences of subsets of $Q^{\mathbf{N}}$ :

$$
\begin{aligned}
& S_{n}(X)=\underbrace{X \times \cdots \times X}_{n \text { times }} \times Q \times Q \times \cdots, \\
& S_{n}^{\prime}(X)=\left\{x \in Q^{\mathbf{N}}: \text { at least } n \text { of the } x_{i} \text { 's are in } X\right\}, \\
& S_{n}^{\prime \prime}(X)=\left\{x \in Q^{\mathbf{N}}: x_{i} \in X \text { for some } i \geq n\right\} .
\end{aligned}
$$

Note that $S_{n}(X) \subset S_{n}^{\prime}(X) \subset S_{n}^{\prime \prime}(X)$ and that $S_{\infty}(X)=X^{\mathbf{N}}$ and $S_{\infty}^{\prime}(X)=S_{\infty}^{\prime \prime}(X)$.

THEOREM 3.1. If $X \subset Q$ is strongly $\mathscr{M}$-universal then the sequences $S(X), S^{\prime}(X)$ and $S^{\prime \prime}(X)$ are strongly $\mathscr{M}$-universal in $Q^{\mathbf{N}}$. If, in addition, $\mathscr{M}$ is closed under finite intersections then $X^{\mathbf{N}}$ and $S_{\infty}^{\prime}(X)$ are strongly $\mathscr{M}_{\delta}$-universal.

Proof. Let $\rho_{n}$ be a metric on $Q$ such that

$$
\rho(x, y)=\max \left\{\rho_{n}\left(x_{n}, y_{n}\right): n \in \mathbf{N}\right\}
$$


is a metric on $Q^{\mathbf{N}}$. Consider a map $f: Q \rightarrow Q^{\mathbf{N}}$ that restricts to a Zembedding on some compactum $K$ and a sequence $Q \supset A_{1} \supset A_{2} \supset$ $\cdots$ of elements of $\mathscr{M}$. We may assume that $f$ is a Z-embedding. Write $Q \backslash K$ as a union of compacta $\left(F_{i}\right)_{i=0}^{\infty}$ with $F_{i} \subset \operatorname{int}\left(F_{i+1}\right)$ and $F_{0}=\varnothing$. Let $\varepsilon>0$ and define the decreasing sequence $\varepsilon_{i}=$ $\min \left\{2^{-i} \varepsilon, \frac{1}{2} \rho\left(f(K), f\left(F_{i}\right)\right)\right\}$. Consider now the $n$-th component $f_{n}: Q \rightarrow Q$ of $f$. We shall construct a sequence $\alpha_{0}, \alpha_{1}, \ldots$ of functions from $Q$ into $Q$ with the following properties:

$$
\begin{gathered}
\rho_{n}\left(\alpha_{i}, \alpha_{i-1}\right)<\varepsilon_{i+1}, \quad \alpha_{i}\left|F_{i-1}=\alpha_{i-1}\right| F_{i-1}, \\
\alpha_{i}\left|Q \backslash F_{i+1}=f_{n}\right| Q \backslash F_{i+1}, \quad \alpha_{i} \mid F_{i} \text { is a Z-embedding }, \\
\alpha_{i}^{-1}(X) \cap F_{i}=A_{n} \cap F_{i} .
\end{gathered}
$$

Put $\alpha_{0}=f_{n}$ and assume that $\alpha_{i}$ has been constructed. Using the strong $\mathscr{M}$-universality of $X$ we find a Z-embedding $\beta: F_{i+1} \rightarrow Q$, close to $\alpha_{i} \mid F_{i+1}$, with $\beta\left|F_{i}=\alpha_{i}\right| F_{i}$ and $\beta^{-1}(X)=A_{n} \cap F_{i+1}$. Extend $\beta$ to a map $\alpha_{i+1}: Q \rightarrow Q$ that restricts to $f$ on $Q \backslash F_{i+2}$.

The $\alpha_{i}$ 's obviously form a Cauchy sequence and we can define the continuous map $g_{n}=\lim _{i \rightarrow \infty} \alpha_{i}$. One may verify that $g_{n}$ has the following properties:

$$
\begin{gathered}
\rho_{n}\left(g_{n}, f_{n}\right)<\varepsilon, \\
\text { if } x \in F_{i+1} \backslash F_{i} \text { then } \rho_{n}\left(g_{n}(x), f_{n}(x)\right)<\rho\left(f(K), f\left(F_{i+1}\right)\right), \\
g_{n}\left|K=f_{n}\right| K,
\end{gathered}
$$

$g_{n} \mid F_{i}$ is a Z-embedding for every $i$,

$$
g_{n}^{-1}(X) \backslash K=A_{n} \backslash K \text {. }
$$

Define $g=\left(g_{n}\right)_{n}: Q \rightarrow Q^{\mathbf{N}}$. Note that $g$ is one-to-one and hence an embedding. The set $g(Q)$ is contained in the $\sigma$ Z-set $f(K) \cup$ $\bigcup_{i=0}^{\infty} g_{1}\left(F_{i}\right) \times Q \times Q \times \cdots$ and is therefore a Z-set. The maps $f$ and $g$ are $\varepsilon$-close and $f|K=g| K$. Let $x \in Q \backslash K$. If $x$ is an element of $A_{n}$ then $x \in \bigcap_{j=1}^{n} A_{j}$. Consequently, we have $g_{j}(x) \in X$ for $j=1,2, \ldots, n$. This means that $g(x) \in S_{n}(X) \subset S_{n}^{\prime}(X) \subset S_{n}^{\prime \prime}(X)$. On the other hand, if $g(x)$ is an element of $S_{n}^{\prime \prime}(X)$ then $g_{j}(x) \in X$ for some $j \geq n$ and hence $x \in A_{j} \subset A_{n}$. This completes the proof.

Consider now the pseudoboundary $B$ of the Hilbert cube. This is an $\mathscr{F}_{\sigma}$-absorber in $Q$. The conditions (1) and (2) of the definition of absorbing system are trivially satisfied by $S(B), S^{\prime}(B)$ and $S^{\prime \prime}(B)$, 
so we have:

Corollary 3.2. The sequences $S(B), S^{\prime}(B)$ and $S^{\prime \prime}(B)$ are $\mathscr{F}_{\sigma}$ absorbing and hence they are homeomorphic in $Q^{\mathbf{N}}$. Moreover, $B^{\mathbf{N}}$ and $S_{\infty}^{\prime}(B)$ are $\mathscr{F}_{\sigma \delta}$-absorbers.

Consider the $\sigma$ Z-set

$$
\sigma=\left\{x \in Q: x_{i}=0 \text { for all but finitely many } i\right\} .
$$

It is well known that $\sigma$ is homeomorphic to $l_{f}^{2}$ and that it is a so-called fd-capset in $Q$ or, in our terminology, an absorber for the strongly countable dimensional $\sigma$-compacta. It is easily verified by juggling coordinates that the system $S(\sigma)$ is homeomorphic to $S(B)$ in $Q^{\mathbf{N}}$ and hence $\mathscr{F}_{\sigma}$-absorbing. Observe that the following systems are all homeomorphic: $S(\sigma)$ in $Q^{\mathbf{N}}, S(\sigma \times I)$ in $(Q \times I)^{\mathbf{N}}, S(\sigma) \times I^{\mathbf{N}}$ in $Q^{\mathbf{N}} \times I^{\mathbf{N}}, S(\sigma) \times Q^{\mathbf{N}}$ in $Q^{\mathbf{N}} \times Q^{\mathbf{N}}, S(\sigma \times Q)$ in $(Q \times Q)^{\mathbf{N}}$ and finally $S(B)$ in $Q^{\mathbf{N}}$.

We can take this one step further:

COROLLARY 3.3. If $Y$ is an $\mathscr{F}_{\sigma \delta}$-absorber in $Q$ then the sequences $S(Y), S^{\prime}(Y)$ and $S^{\prime \prime}(Y)$ are $\mathscr{F}_{\sigma \delta}$-absorbing and hence they are homeomorphic in $Q^{\mathbf{N}}$. Moreover, $Y^{\mathbf{N}}$ and $S_{\infty}^{\prime}(Y)$ are also $\mathscr{F}_{\sigma \delta}$-absorbers.

4. The space of infinite-dimensional compacta. In this section we prove Theorem 1.1. The following lemma is easily verified.

LEMMA 4.1. If $X$ and $Y$ are compact spaces and if $F: X \rightarrow 2^{Y}$ is continuous then $G(A)=\bigcup\{F(a): a \in A\}$ defines a continuous map from $2^{X}$ into $2^{Y}$.

Proposition 4.2. The sequence $\left(\operatorname{Dim}_{\geq k}\right)_{k=1}^{\infty}$ is reflexively universal in $2 Q$.

Proof. Let $F: 2^{Q} \rightarrow 2^{Q}$ be a map and let $K$ be a closed subset of $2^{Q}$ such that $F \mid K$ is a Z-embedding. We may assume that $F$ is a Z-embedding. Let $\varepsilon: 2^{Q} \rightarrow I$ be a map with the properties: $\varepsilon^{-1}(0)=$ $F(K)$ and $\varepsilon(A) \leq d(A, F(K)) / 4$ for each $A \in 2^{Q}$. According to Curtis [5] the finite sets in $2^{Q}$ contain an fd-capset and hence there exists a deformation $H_{t}$ of $2^{Q}$ such that $H_{0}=1$ and $H_{t}(A)$ is finite for $t>0$ and $A \in 2^{Q}$. We may assume, moreover, that $d\left(H_{t}, 1\right) \leq 2 t$ and that $H_{t}(A) \subset[0,1-t]^{\mathbf{N}}$ for every $t$ and $A$. 
We shall use the vector addition and scalar multiplication operations that $Q$ inherits from $\mathbf{R}^{\mathbf{N}}$. Define the homotopy $\alpha_{t}: 2^{Q} \rightarrow 2^{Q}$ by

$$
\alpha_{t}(A)=\{0\} \cup \bigcup_{n=1}^{\infty}\left\{\frac{t}{n}\right\} \times \frac{t}{n} \vec{A},
$$

where $\vec{A}$ is the subset of $\prod_{i=2}^{\infty} I$ that is obtained from $A$ by a coordinate shift. Note that $\alpha_{t}(A) \subset[0, t]^{\mathbf{N}}$ and that $\alpha_{0}(A)=\{0\}$. The map $G: 2^{Q} \rightarrow 2^{Q}$ that approximates $F$ is defined by

$$
G(A)=H_{\varepsilon(F(A))}(F(A))+\alpha_{\varepsilon(F(A))}(A) .
$$

The function $G$ is continuous by Lemma 4.1 and the continuity of the homotopies $H$ and $\alpha$. Observe that $d(G(A), F(A)) \leq 3 \varepsilon(F(A))$ for every $A \in 2^{Q}$. If $A \in K$ then $\varepsilon(F(A))=0$ and hence $G$ restricts to $F$ on $K$. Let $A$ be an element of $2^{Q} \backslash K$. Then $t=\varepsilon(F(A))>0$ and hence $H_{t}(F(A))$ is finite. So $G(A)$ is a finite union of translates of $\alpha_{t}(A)$ and consequently a union of a finite set and a countable collection of copies of $A$. This means that $G$ preserves dimension and

$$
G^{-1}\left(\operatorname{Dim}_{\geq k}\right) \backslash K=\operatorname{Dim}_{\geq k} \backslash K .
$$

We shall now show that $G$ is one-to-one. The restriction of $G$ to $K$ is obviously one-to-one. If $A \in 2^{Q} \backslash K$ then $d(G(A), F(A)) \leq$ $3 \varepsilon(F(A))<d(F(K), F(A))$ and hence $G(A)$ is not in $G(K)=F(K)$. For the remaining case let $A, B \in 2^{Q} \backslash K$ such that $G(A)=G(B)$. Let $\pi: Q \rightarrow I$ be the projection onto the first coordinate and define the positive numbers $r=\varepsilon(F(A))$ and $t=\varepsilon(F(B))$. Select a point $y=$ $(a, x) \in G(A)=G(B)$ such that $a=\min (\pi(G(A)))=\min (\pi(G(B)))$. Note that $y$ is an element of both $H_{r}(F(A))$ and $H_{t}(F(B))$. Since the latter sets are finite we can define $\lambda>0$ as one half of the distance of $y$ towards the other points in $H_{r}(F(A)) \cup H_{t}(F(B))$.

Let $m$ and $n$ be the first numbers that satisfy $\frac{r}{m} \leq \lambda$ and $\frac{t}{n} \leq \lambda$. We now have:

$$
\begin{aligned}
(\{y\} & \left.+[0, \lambda]^{\mathbf{N}}\right) \cap G(A)=\{y\} \cup \bigcup_{i=m}^{\infty}\left\{a+\frac{r}{i}\right\} \times\left(x+\frac{r}{i} \vec{A}\right) \\
& =\left(\{y\}+[0, \lambda]^{\mathbf{N}}\right) \cap G(B)=\{y\} \cup \bigcup_{i=n}^{\infty}\left\{a+\frac{t}{i}\right\} \times\left(x+\frac{t}{i} \vec{B}\right) .
\end{aligned}
$$

This implies:

$$
\left\{a+\frac{r}{m}\right\} \times\left(x+\frac{r}{m} \vec{A}\right)=\left\{a+\frac{t}{n}\right\} \times\left(x+\frac{t}{n} \vec{B}\right) .
$$


This means that $\frac{r}{m}=\frac{t}{n}$ and $\frac{r}{m} \vec{A}=\frac{t}{n} \vec{B}$ and hence that $A=B$. So $G$ is one-to-one and therefore an embedding.

Observe that $\pi(G(A))$ is countable if $A \in 2^{Q} \backslash K$ so $G(A)$ is nowhere dense in $Q$. Since $D_{t}(A)=\{x \in Q: d(x, A) \leq t\}$ is a deformation of $Q$ through the complement of $G\left(2^{Q} \backslash K\right)$, we have that $G\left(2^{Q} \backslash K\right)$ is a $\sigma$ Z-set. Consequently, $G\left(2^{Q}\right) \subset F(K) \cup G\left(2^{Q} \backslash K\right)$ is a $\mathrm{Z}$-set and $G$ is a Z-embedding. This completes the proof.

Observing that $G$ preserves many other properties we find for instance:

COROLlaRY 4.3. The sequence $\left(\overline{\mathrm{Dim}}_{\geq k}\right)_{k=1}^{\infty}$ is reflexively universal in $2^{Q}$.

COROLLARY 4.4. The sequence consisting of the collections of compacta of cohomological dimension not less than $k$ is reflexively universal in $2^{Q}$.

Corollary 4.5. The transfinite sequence $\left\{A \in 2^{Q}: \operatorname{ind}(A) \geq \alpha\right\}_{\alpha<\omega_{1}}$ is reflexively universal in $2^{Q}$.

THEOREM 4.6. The sequence $\left(\operatorname{Dim}_{\geq k}\right)_{k=1}^{\infty}$ is $\mathscr{F}_{\sigma}$-absorbing in $2^{Q}$. Consequently, $\operatorname{Dim}_{\infty}$ is an $\mathscr{F}_{\sigma \delta}$-absorber.

Proof. Let $k, n \in \mathbf{N}$ and define

$\mathscr{G}_{n}=\left\{A \in 2^{Q}:\right.$ there is in $Q$ a finite open cover of $A$ with mesh $\leq 1 / n$ and order $\leq k\}$.

Obviously, $\mathscr{G}_{n}$ is an open subset of $2 Q$. Note that $\operatorname{Dim}_{\geq k}=$ $Q \backslash \bigcap_{n=1}^{\infty} \mathscr{G}_{n}$ is therefore an $F_{\sigma}$-set. According to Curtis [5] the finite sets in $2 Q$ contain an fd-capset and hence $\operatorname{Dim}_{\geq 1}$ is a $\sigma$ Z-set.

In view of Proposition 4.2 it suffices to show that the system is $\mathscr{F}_{\sigma^{-}}$ universal. The space $\operatorname{Dim}_{1}(I)$ is an $\mathscr{F}_{\sigma}$-absorber in the Hilbert cube $2^{I}$. This can be found essentially in Kroonenberg [10] if we note that $H_{t}(A)=\{x \in I: d(x, A) \leq t\}$ is a deformation of $2^{I}$ through $\operatorname{Dim}_{1}(I)$, see also [1]. So the pair $\left(2^{I}, \operatorname{Dim}_{1}(I)\right)$ is homeomorphic to $(Q, B)$. Corollary 3.2 now guarantees that $S^{\prime}\left(\operatorname{Dim}_{1}(I)\right)$ is an $\mathscr{F}_{\sigma^{-}}$ absorbing sequence in $\left(2^{I}\right)^{\mathbf{N}}$. Define the embedding $\alpha:\left(2^{I}\right)^{\mathbf{N}} \rightarrow 2^{Q}$ by $\alpha\left(\left(P_{i}\right)_{i=1}^{\infty}\right)=\prod_{i=1}^{\infty} P_{i}$. Since $\prod_{i=1}^{\infty} P_{i}$ is $k$-dimensional if and only if precisely $k$ of the $P_{i}$ 's are in $\operatorname{Dim}_{1}(I)$, we have

$$
\alpha^{-1}\left(\operatorname{Dim}_{\geq k}\right)=S_{k}^{\prime}\left(\operatorname{Dim}_{1}(I)\right) \text {. }
$$


The sequence $\operatorname{Dim}_{\geq k}$ is then $\mathscr{F}_{\sigma}$-universal because $S^{\prime}\left(\operatorname{Dim}_{1}(I)\right)$ is.

We find Theorem 1.1 by combining Theorem 2.1, Corollary 3.2 and Theorem 4.6. The fact that $\left(2^{Q},\left(\operatorname{Dim}_{\geq k}\right)_{k=1}^{\infty}\right)$ is homeomorphic to $\left(Q^{\mathbf{N}}, S(B)\right)$ means that there exists a homeomorphism $\alpha: 2^{Q} \rightarrow Q^{\mathbf{N}}$ such that

$$
\alpha\left(\operatorname{Dim}_{\geq k}\right)=\underbrace{B \times \cdots \times B}_{k \text { times }} \times Q \times Q \times \cdots .
$$

This implies that $\alpha\left(\operatorname{Dim}_{\infty}\right)=B^{\mathbf{N}}$, which space is homeomorphic to $\left(l_{f}^{2}\right)^{\omega}$. Observe that in view of the remark following Corollary $3.2 \mathrm{it}$ is also possible to find an $\alpha^{\prime}$ with

$$
\alpha^{\prime}\left(\operatorname{Dim}_{\geq k}\right)=\underbrace{\sigma \times \cdots \times \sigma}_{k \text { times }} \times Q \times Q \times \cdots .
$$

Comparing $\left(2^{Q}, \operatorname{Dim}_{\geq k}\right)$ with $\left(Q^{\mathbf{N}}, S^{\prime \prime}(B)\right)$ we find part (b) of Theorem 1.1. There exists a homeomorphism $\beta$ from $2^{Q}$ onto $Q^{\mathbf{N}}$ such that for every $k \in\{0,1,2, \ldots\}$,

$$
\beta\left(\operatorname{Dim}_{\leq k}\right)=\underbrace{Q \times \cdots \times Q}_{k \text { times }} \times s \times s \times \cdots .
$$

Note that

$$
\beta\left(\operatorname{Dim}_{k}\right)=\underbrace{Q \times \cdots \times Q}_{k-1 \text { times }} \times B \times s \times s \times \cdots
$$

and hence the pair $\left(\operatorname{Dim}_{\leq k}, \operatorname{Dim}_{k}\right), 0<k<\infty$, is homeomorphic to $(Q \times s, B \times s)$, i.e., $\operatorname{Dim}_{k}$ is a so-called Z-absorber in the topological Hilbert space $\operatorname{Dim}_{\leq k}$.

Let $\mathrm{cDim}_{\geq k}$ stand for all elements of $2^{Q}$ with cohomological dimension at least $k$ with respect to for instance the group $\mathbf{Z}$.

\section{QUESTION. Is $\mathrm{cDim}_{\geq k} \sigma$-compact?}

Observe that it follows from the proof of Theorem 4.6 that the sequence $\mathrm{cDim}_{\geq k}$ is $\mathscr{F}_{\sigma}$-universal. If the answer to the question is yes then we have in view of Corollary 4.4 and the fact $\mathrm{cDim}_{\geq 1}=\operatorname{Dim}_{\geq 1}$ that $\mathrm{cDim}_{\geq k}$ is $\mathscr{F}_{\sigma}$-absorbing and $\mathrm{cDim}_{\infty}$ is homeomorphic to $B^{\overline{\mathbf{N}}}$.

5. Uniformly $\geq k$-dimensional compacta in $2^{Q}$. This section is devoted to the proof of Theorem 1.2. Consider the following decreasing sequence of subsets of $\left(2^{Q}\right)^{\mathbf{N}}$ :

$$
X_{k}=\left\{P \in\left(2^{Q}\right)^{\mathbf{N}}: P_{i} \in \operatorname{Dim}_{\geq k} \text { for infinitely many } i\right\} .
$$


LEMMA 5.1. The sequence $\left(X_{k}\right)_{k=1}^{\infty}$ is $\mathscr{F}_{\sigma \delta}$-universal.

Proof. Let $A_{1} \supset A_{2} \supset \cdots$ be a sequence of $F_{\sigma \delta}$-sets in $Q$. Choose $\sigma$-compact sets $A_{k}^{n}$ such that $A_{k}^{n+1} \cup A_{k+1}^{n} \subset A_{k}^{n}$ and $A_{k}=\bigcap_{n=1}^{\infty} A_{k}^{n}$. Since $\left(\operatorname{Dim}_{\geq k}\right)_{k=1}^{\infty}$ is $\mathscr{F}_{\sigma}$-universal, Theorem 4.6 , there exist embeddings $f_{n}: Q \rightarrow 2^{Q}$ such that $f_{n}^{-1}\left(\operatorname{Dim}_{\geq k}\right)=A_{k}^{n}$. Put $f=\left(f_{n}\right)_{n}: Q \rightarrow$ $\left(2^{Q}\right)^{\mathbf{N}}$. If $x \in A_{k}$ then $x \in A_{k}^{n}$ for all $n$. So $f_{n}(x) \in \operatorname{Dim}_{\geq k}$ for all $n$ and hence $f(x) \in X_{k}$. If $x \notin A_{k}$ then $x \notin A_{k}^{j}$ for some $j$, so $x \notin A_{k}^{n}$ for all $n \geq j$. Consequently, $f_{n}(x) \notin \operatorname{Dim}_{\geq k}$ for all $n \geq j$ and $f(x) \notin X_{k}$.

REMARK. One may use the method of Theorem 3.1 to show that $\left(X_{k}\right)_{k}$ is in fact $\mathscr{F}_{\sigma \delta}$-absorbing in $\left(2^{Q}\right)^{\mathbf{N}}$.

Proposition 5.2. The sequence $\left(\overline{\operatorname{Dim}}_{\geq k}\right)_{k=1}^{\infty}$ is strongly $\mathscr{F}_{\sigma \delta}$-universal.

Proof. In view of Corollary 4.3 it suffices to show that the sequence is $\mathscr{F}_{\sigma \delta}$-universal. We shall prove that the system $X_{k}$ can be embedded in $\overline{\operatorname{Dim}}_{\geq k}$.

Let $G$ stand for the compact, multiplicative subspace $\{0\} \cup$ $\left\{2^{-m}: m=1,2, \ldots\right\}$ of $I$. According to Curtis [5] there exists a deformation $H_{t}: 2^{Q} \rightarrow 2^{Q}$ such that $H_{0}=1$ and $H_{t}(A)$ is finite if $t>0$. Let $P=\left(P_{m}\right)_{m=1}^{\infty}$ be an element of $\left(2^{Q}\right)^{\mathbf{N}}$. We define the continuous function $F: G \times\left(2^{Q}\right)^{\mathbf{N}} \rightarrow 2^{Q}$ by

$$
F_{0}(P)=\{0\} \quad \text { and } \quad F_{2^{-m}}(P)=2^{-m} P_{m} \cup\{0\} .
$$

We shall define inductively a sequence of compacta $\left(A_{n}\right)_{n=1}^{\infty}$ such that

$$
A_{n} \subset(G \times Q)^{n-1} \times G,
$$

i.e., the $n$ odd coordinates are in $G$ and the $n-1$ even ones in $Q$. Put $A_{1}(P)=G$ and

$$
\begin{aligned}
& A_{n+1}(P)=\bigcup\left\{\{(x, a)\} \times H_{a b}\left(F_{a}(P)\right) \times\{a b\}:\right. \\
&\left.(x, a) \in A_{n}(P) \text { and } b \in G\right\} .
\end{aligned}
$$

Here $(x, a) \in A_{n}$ means that $x \in(G \times Q)^{n-1}$ and $a \in G$. Note that since $a b<a$ the odd components of the points in $A_{n}$ form a decreasing sequence. Applying Lemma 4.1 we find that every $A_{n}$ is a compactum that depends continuously on $P$. We identify each $A_{n}$ with its copy $A_{n} \times\{(0,0, \ldots)\}$ in $(G \times Q)^{\mathbf{N}} \subset(I \times Q)^{\mathbf{N}}$. The Hilbert 
cube $Q^{\prime}=(I \times Q)^{\mathbf{N}}$ is equipped with the metric $\rho=\max _{i \in \mathbf{N}} \rho_{i}$, where $\rho_{2 j-1}$ is a standard metric on $I$ that is bounded by $2^{-2 j+1}$ and $\rho_{2 j}$ is a standard metric on $Q$ that is bounded by $2^{-2 j}$. Observe that $\pi_{n}\left(A_{n+1}\right)=A_{n}$, where $\pi_{n}$ is the projection from $Q^{\prime}$ onto $(I \times Q)^{n-1} \times$ $I$. This implies that $\rho\left(\pi_{n}, 1\right) \leq 2^{-2 n}$ and $\rho\left(A_{n}, A_{n+1}\right) \leq 2^{-2 n}$ so that $\left(A_{n}(P)\right)_{n=1}^{\infty}$ is a Cauchy sequence of maps. So $\alpha(P)=\lim _{n \rightarrow \infty} A_{n}(P)$ defines a continuous map from $\left(2^{Q}\right)^{\mathbf{N}}$ into $2^{Q^{\prime}}$. In addition, we find that $\alpha(P)=\bigcap_{n=1}^{\infty} \pi_{n}^{-1}\left(A_{n}\right)$. Since 0 is an element of every $F_{t}(P)$ we have $A_{n} \subset A_{n+1}$. This implies that $\alpha(P)$ is the closure of $Y=$ $\bigcup_{n=1}^{\infty} A_{n}$ in $Q^{\prime}$.

We show by induction that

$$
A_{n}^{\prime}=\left\{(x, a) \in A_{n}: a \neq 0\right\}
$$

is countable. This is obviously true for $A_{1}^{\prime}$. Let $(x, a, p, a b)$ be an element of $A_{n+1}^{\prime}$. So $a b \neq 0,(x, a) \in A_{n}$ and $p \in H_{a b}\left(F_{a}(P)\right)$. This implies $a \neq 0$ and $(x, a) \in A_{n}^{\prime}$ and hence we have:

$$
\begin{aligned}
& A_{n+1}^{\prime}=\bigcup\left\{\{(x, a)\} \times H_{a b}\left(F_{a}(P)\right) \times\{a b\}:\right. \\
& \left.\qquad(x, a) \in A_{n}^{\prime} \text { and } b \in G \backslash\{0\}\right\} .
\end{aligned}
$$

This is a countable union of finite sets because $H_{a b}\left(F_{a}(P)\right)$ is finite if $a b \neq 0$. Consequently, the set $A_{n+1}^{\prime}$ is countable.

Assume that $P \notin X_{k}$. We shall prove that 0 has a neighbourhood in $\alpha(P)$ with dimension less than $k$. Since $P \notin X_{k}$ there exists an $m$ such that $\operatorname{dim}\left(P_{i}\right)<k$ for all $i \geq m$. So if we put $c=2^{-m}$ then $\operatorname{dim}\left(F_{a}(P)\right)<k$ for $a \leq c$. Let $C$ consist of all points in $Q^{\prime}$ whose first component is less than or equal to $c$. We shall prove inductively that $\operatorname{dim}\left(A_{n} \cap C\right)<k$. Obviously, we have $\operatorname{dim}\left(A_{1} \cap C\right)=0$. Assume that $\operatorname{dim}\left(A_{n} \cap C\right)<k$ and consider

$$
A_{n+1} \cap C=\bigcup\left\{\{(x, a)\} \times H_{a b}\left(F_{a}(P)\right) \times\{a b\}:\right.
$$

$$
\left.(x, a) \in A_{n} \cap C \text { and } b \in G\right\} .
$$

If $a=0$ then $a b=0$ and $H_{a b}\left(F_{a}(P)\right)=\{0\}$. Consequently, we have:

$$
\begin{aligned}
A_{n+1} \cap C=\left(A_{n} \cap C\right) \cup \bigcup\left\{\{(x, a)\} \times H_{a b}\left(F_{a}(P)\right) \times\{a b\}:\right. \\
\left.(x, a) \in A_{n}^{\prime} \cap C \text { and } b \in G\right\} .
\end{aligned}
$$

Note that the $H_{a b}\left(F_{a}(P)\right)$ in this expression is either finite or homeomorphic to $F_{a}(P)$. Since the odd components of points form a decreasing sequence in $G$ we have that $a \leq c$ whenever $(x, a)$ is a 
point in $A_{n} \cap C$. So every $F_{a}(P)$ is less than $k$-dimensional. Since $A_{n}^{\prime}$ is countable, the set $A_{n+1} \cap C$ is a countable union of $<k$ dimensional compacta and therefore $\operatorname{dim}\left(A_{n+1} \cap C\right)<k$. Note that $\alpha(P) \cap C=\bigcap_{n=1}^{\infty} \pi_{n}^{-1}\left(A_{n} \cap C\right)$. Since $\pi_{n}^{-1}\left(A_{n} \cap C\right)$ is the product of a $<k$-dimensional compactum and a Hilbert cube of diameter $\leq 2^{-2 n}$, there is for every $n$ an open cover of $\pi_{n}^{-1}\left(A_{n} \cap C\right.$ ) (and hence of $\alpha(P) \cap C)$ with mesh $\leq 2^{-2 n}$ and order $\leq k$. Consequently, we have $\operatorname{dim}(\alpha(P) \cap C)<k$ and

$$
\alpha(P) \notin \overline{\operatorname{Dim}}_{\geq k}\left(Q^{\prime}\right) .
$$

Consider now the case $P \in X_{k}$. This means that $\operatorname{dim}\left(F_{a}(P)\right) \geq k$ for infinitely many $a \in G$. Let $(x, 0) \in A_{n}$. We show by induction that $A_{n+1}$ is at least $k$-dimensional at this point, i.e., every neighbourhood of the point in $A_{n+1}$ has dimension no less than $k$. First, consider $0 \in A_{1}$. We have:

$$
A_{2}=\bigcup_{a, b \in G}\{a\} \times H_{a b}\left(F_{a}(P)\right) \times\{a b\} .
$$

Selecting $b=0$ we find

$$
\lim _{a \rightarrow 0}\{a\} \times H_{0}\left(F_{a}(P)\right) \times\{0\}=\lim _{a \rightarrow 0}\{a\} \times F_{a}(P) \times\{0\}=\{0\}
$$

and hence $A_{2}$ is $\geq k$-dimensional at 0 .

Assume that the induction hypothesis is valid for points $(x, 0)$ in $A_{n}$. If $(y, 0) \in A_{n+1}$ then $y=(x, a, p)$, where $(x, a) \in A_{n}$ and $p \in H_{0}\left(F_{a}(P)\right)=F_{a}(P)$. If $a=0$ then $F_{a}(P)=\{0\}$ and $p=0$. This means that $(y, 0)=(x, 0,0,0) \in A_{n}$ and by induction $A_{n+1}$ and therefore $A_{n+2}$ are $\geq k$-dimensional at the point. If $a \neq 0$ then for $b, c \in G$ we have:

$$
\{(x, a)\} \times H_{a b}\left(F_{a}\right) \times\{a b\} \times H_{a b c}\left(F_{a b}\right) \times\{a b c\} \subset A_{n+2},
$$

where we denote $F_{a}(P)$ simply by $F_{a}$. Since $\lim _{b \rightarrow 0} H_{a b}\left(F_{a}\right)=$ $H_{0}\left(F_{a}\right)=F_{a}$ in $2^{Q}$ we can find points $p_{b} \in H_{a b}\left(F_{a}\right)$ such that $\lim _{b \rightarrow 0} p_{b}=p$. Selecting $c=0$ we find

$$
\lim _{b \rightarrow 0}\left\{\left(x, a, p_{b}, a b\right)\right\} \times F_{a b} \times\{0\}=\{(x, a, p, 0,0,0)\} .
$$

Since $F_{a b}$ is $\geq k$-dimensional for infinitely many $b$ 's we have that $A_{n+2}$ is $\geq k$-dimensional at $(y, 0,0, \ldots)=(x, a, p, 0,0, \ldots)$. This completes the induction.

If $x$ is an element of $A_{n}$ then $(x, 0,0)$ is in $A_{n+1}$ and hence $A_{n+2}$ is $\geq k$-dimensional at $x$. Consequently, the set $Y=\bigcup_{n=1}^{\infty} A_{n}$ 
is $\geq k$-dimensional at each of its points. So its closure $\alpha(P)$ is an element of $\overline{\operatorname{Dim}}_{\geq k}\left(Q^{\prime}\right)$ and we have:

$$
\alpha^{-1}\left(\overline{\operatorname{Dim}}_{\geq k}\left(Q^{\prime}\right)\right)=X_{k} \text {. }
$$

This does not quite complete the proof of Proposition 5.2 since $\alpha$ is not one-to-one. This can easily be fixed, however. Define the map $\beta$ from $\left(2^{Q}\right)^{\mathbf{N}}$ into the hyperspace of $Q^{\prime \prime}=I \times Q^{\prime} \times \prod_{i=1}^{\infty} Q$ by

$$
\beta(P)=(\{0\} \times \alpha(P) \times\{(0,0, \ldots)\}) \cup\left(\{1\} \times Q^{\prime} \times \prod_{i=1}^{\infty} P_{i}\right) .
$$

The map $\beta$ is obviously one-to-one and hence an embedding. Note that $\beta(P)$ is a topological sum of a copy of $\alpha(P)$ and a uniformly infinite-dimensional space, so we retain the property

$$
\beta^{-1}\left(\overline{\operatorname{Dim}}_{\geq k}\left(Q^{\prime \prime}\right)\right)=X_{k} .
$$

We may conclude that $\left(\overline{\operatorname{Dim}}_{\geq k}\left(Q^{\prime \prime}\right)\right)_{k=1}^{\infty}$ is $\mathscr{F}_{\sigma \delta}$-universal just as $\left(X_{k}\right)_{k=1}^{\infty}$.

THEOREM 5.3. The sequence $\left(\overline{\operatorname{Dim}}_{\geq k}\right)_{k=1}^{\infty}$ is $\mathscr{F}_{\sigma \delta}$-absorbing and $\overline{\operatorname{Dim}}_{\infty}$ is an $\mathscr{F}_{\sigma \delta}$-absorber in $2 Q$.

Proof. Note that $\overline{\operatorname{Dim}}_{\geq 1}$ is contained in the $\sigma$ Z-set $\operatorname{Dim}_{\geq 1}$. It remains to be shown that every $\overline{\operatorname{Dim}}_{\geq k}$ is in $\mathscr{F}_{\sigma \delta}$. Let $\left\{O_{i}: i \in \mathbf{N}\right\}$ be a countable open basis for the topology of $Q$ and let $k \in \mathbf{N}$. Write every $O_{i}$ as a countable union of compacta $F_{i}^{1} \subset F_{i}^{2} \subset \cdots$. Define the collections

$\mathscr{G}_{i}^{j}=\left\{A \in 2^{Q}:\right.$ there is in $Q$ an finite open cover $\mathscr{U}$ of $A \cap F_{i}^{j}$ with mesh $\leq 1 / j$ and order $\leq k\}$.

If $A \in \mathscr{G}_{i}^{j}$ and $\mathscr{U}$ is such a cover then put $\varepsilon=\rho\left(A, F_{i}^{j} \backslash \bigcup \mathscr{U}\right)$. Observe that if $\rho(A, B)<\varepsilon$ then $B \cap F_{i}^{j}$ is also covered by $\mathscr{U}$ and hence $\mathscr{G}_{i}^{j}$ is open in $2^{Q}$. So $\mathscr{G}_{i}=\bigcap_{j=1}^{\infty} \mathscr{G}_{i}^{j}$ is a $G_{\delta}$-set. Since a countable union of $<k$-dimensional compacta is again $<k$-dimensional one easily verifies that an element $A$ of $2^{Q}$ is in $\mathscr{G}_{i}$ if and only if $\operatorname{dim}\left(A \cap O_{i}\right)<k$. The collection $\mathscr{G}_{i}^{\prime}=\mathscr{G}_{i} \backslash\left\{A \in 2^{Q}: A \cap O_{i}=\varnothing\right\}$ is obviously also $G_{\delta}$. Observe that $\bigcup_{i=1}^{\infty} \mathscr{G}_{i}^{\prime}$ is precisely the complement of $\overline{\operatorname{Dim}}_{\geq k}$ in $2^{Q}$. This shows that $\overline{\operatorname{Dim}}_{\geq k}$ is in $\mathscr{F}_{\sigma \delta}$.

We find Theorem 1.2 by combining Theorem 2.1, Corollary 3.3 and Theorem 5.3. If $Y$ is an $\mathscr{F}_{\sigma \delta}$-absorber in $Q$ then there exists 
a homeomorphism $\alpha$ from $2^{Q}$ onto $Q^{\mathbf{N}}$ such that for every $k \in$ $\{0,1,2, \ldots\}$,

$$
\alpha\left(\overline{\operatorname{Dim}}_{\geq k}\right)=\underbrace{Y \times \cdots \times Y}_{k \text { times }} \times Q \times Q \times \cdots .
$$

Note that $\overline{\operatorname{Dim}}_{\geq k}, 0<k \leq \infty$, is an $\mathscr{F}_{\sigma \delta}$-absorber and hence homeomorphic to $B^{\overline{\mathbf{N}}}$ and $\left(l_{f}^{2}\right)^{\omega}$.

6. Function spaces in the topology of pointwise convergence. In this section the Hilbert cube $Q$ is represented by $\widehat{\mathbf{R}}^{\mathbf{N}}$, where $\widehat{\mathbf{R}}$ stands for the compactification $[-\infty, \infty]$. Consequently, $\mathbf{R}^{\mathbf{N}}$ is the pseudointerior of $Q$. If $X$ is countable metric space then $C_{p}(X)$ denotes the space of continuous, realvalued functions on $X$ endowed with the topology of pointwise convergence. Define the following subspaces of $\mathbf{R}^{\mathbf{N}}$ :

$$
c_{0}=\left\{x \in \mathbf{R}^{\mathbf{N}}: \lim _{i \rightarrow \infty} x_{i}=0\right\}
$$

and for $n \in \mathbf{N}$

$$
\Sigma_{n}=\left\{x \in \mathbf{R}^{\mathbf{N}}:\left|x_{i}\right| \leq 2^{-n} \text { for all but finitely many } i\right\} .
$$

Observe that $\Sigma=\left(\Sigma_{n}\right)_{n}$ is a decreasing sequence of $\sigma$ Z-sets in $Q$ with the property that its intersection is $c_{0}$. The aim of this section is to show that $c_{0}$ and $C_{p}(X)$ are $\mathscr{F}_{\sigma \delta}$-absorbers in the Hilbert cubes $\widehat{\mathbf{R}}^{\mathbf{N}}$ respectively $\widehat{\mathbf{R}}^{X}$. This is an improvement over the result of Dobrowolski, Gul'ko and Mogilski [8] and, independently, Cauty [3] that $c_{0}$ and $C_{p}(X)$ are homeomorphic to $\left(l_{f}^{2}\right)^{\omega}$.

\section{Proposition 6.1. The system $\Sigma$ is $\mathscr{F}_{\sigma}$-universal in $Q$.}

Proof. We shall use the following fact: if $A$ is an $\mathscr{F}_{\sigma}$-absorber in $Q$ and $A^{\prime}$ is a $\sigma \mathrm{Z}$-set then for every $\sigma$-compactum $C$ in $Q$ there is an embedding $f: Q \rightarrow Q$ such that $f^{-1}(A)=C$ and $f(Q \backslash C) \cap A^{\prime}=$ $\varnothing$. This can be seen as follows. The proof of Theorem 2.1 shows that if $A_{1} \supset A_{2}$ is an $\mathscr{F}_{\sigma}$-absorbing system in $Q$ then there is a homeomorphism $h: Q \rightarrow Q$ such that $h(A)=A_{2}$ and $h\left(A^{\prime}\right) \subset A_{1}$. Such a system exists by Corollary 3.2 and it has the required property.

Let $A_{1} \supset A_{2} \supset \cdots$ be a sequence of $\sigma$-compacta in $Q$. Let $\alpha$ be a bijection from $\mathbf{N} \times \mathbf{N}$ onto $\mathbf{N}$ and define $N_{i}=\{\alpha(i, j): j \in \mathbf{N}\}$. For every $i \in \mathbf{N}$ define the Hilbert cube $Q_{i}=\left[-2^{-i+1}, 2^{-i+1}\right]^{N_{i}}$. It is easily verified with the capset characterization theorem in Curtis [4] that

$$
C_{i}=\left\{x \in Q_{i}:\left|x_{\alpha(i, j)}\right| \leq 2^{k-j} \text { for some } k\right\}
$$


is an $\mathscr{F}_{\sigma}$-absorber in $Q_{i}$. Observe that for every $x \in C_{i}$ we have $\lim _{j \rightarrow \infty} x_{\alpha(i, j)}=0$. Define in $Q_{i}$ the $\sigma$ Z-set

$$
D_{i}=\left\{x \in Q_{i}:\left|x_{\alpha(i, j)}\right| \leq 2^{-i} \text { for all but finitely many } j\right\} .
$$

Let $f_{i}: Q \rightarrow Q_{i}$ be an embedding such that $f_{i}^{-1}\left(C_{i}\right)=A_{i}$ and $f_{i}\left(Q \backslash A_{i}\right)$ does not meet $D_{i}$. Consider the embedding $f=$ $\left(f_{i}\right)_{i \in \mathbf{N}}: Q \rightarrow \prod_{i=1}^{\infty} Q_{i} \subset Q$. Let $x \in A_{n}$. If $i>n$ then we have $f_{i}(x) \in Q_{i}$ and hence all components of $f_{i}(x)$ are in $\left[-2^{-n}, 2^{-n}\right]$. If $i \leq n$ then we have $x \in A_{i}$ and hence $f_{i}(x) \in C_{i}$. Note that only finitely many components of $f_{i}(x)$ are outside $\left[-2^{-n}, 2^{-n}\right]$ and hence only finitely many components of $f(x)$ are outside this interval. This means that $f(x)$ is an element of $\Sigma_{n}$. If $x \notin A_{n}$ then we have $f_{n}(x) \notin D_{n}$. This means that infinitely many components of $f_{n}(x)$ have absolute value greater than $2^{-n}$ and hence $f(x) \notin \Sigma_{n}$. So we may conclude that $f^{-1}\left(\Sigma_{n}\right)=A_{n}$.

A subset $A$ is locally homotopy negligible in $X$ if for every map $f: M \rightarrow X$ from an absolute neighbourhood retract $M$ and for every open cover $\mathscr{U}$ of $X$ there exists a homotopy $h: M \times[0,1] \rightarrow X$ such that $\{h(\{x\} \times[0,1])\}_{x \in M}$ refines $\mathscr{U}, h(x, 0)=f(x)$ and $h(M \times(0,1]) \subset X \backslash A$. According to Theorem 2.4 in Toruńczyk [13] $A$ is locally homotopy negligible if the above condition is satisfied for $M=Q$.

For a space $X$ and $* \in X$ we define the weak cartesian product

$$
W(X, *)=\left\{x \in X^{\mathbf{N}}: x_{i}=* \text { for all but finitely many } i\right\} .
$$

Let $\Gamma$ be an ordered set. The following lemma is an adaptation to our needs of Proposition 3.2 in Dobrowolski, Gul'ko and Mogilski [8].

LEMMA 6.2. Let $X=\left(X_{\gamma}\right)_{\gamma \in \Gamma}$ be an order preserving system in $Q$ such that $Q \backslash \bigcap_{\gamma \in \Gamma} X_{\gamma}$ is locally homotopy negligible in $Q$ and let $* \in \bigcap_{\gamma \in \Gamma} X_{\gamma}$. Assume that there exists a homeomorphism $\Phi: Q \rightarrow Q^{\mathbf{N}}$ satisfying

$$
W\left(X_{\gamma}, *\right) \subset \Phi\left(X_{\gamma}\right) \subset X_{\gamma}^{\mathbf{N}}
$$

for all $\gamma \in \Gamma$. Then $X$ is reflexively universal.

Proof. Let $f: Q \rightarrow Q$ be a map that restricts to a Z-embedding on some compact set $K$ and let $\varepsilon: Q \rightarrow(0,1)$ be a continuous function. We can assume that $f(Q \backslash K) \subset \bigcap_{\gamma \in \Gamma} X_{\gamma} \backslash f(K)$. We choose a metric $d$ on $Q^{\mathbf{N}}$ so that $d\left(x, x^{\prime}\right) \leq 2^{-k-2}$ if $x$ and $x^{\prime}$ agree on the first $k$ 
coordinates. Let $\varepsilon^{\prime}: Q^{\mathbf{N}} \rightarrow(0,1)$ be a Lipschitz function such that if maps $f_{1}, f_{2}: Q \rightarrow Q^{\mathbf{N}}$ are $\varepsilon^{\prime}$-close, then $\Phi^{-1} \circ f_{1}$ and $\Phi^{-1} \circ f_{2}$ are $\varepsilon$ close. Define $\delta: Q^{\mathbf{N}} \rightarrow[0,1)$ by $\delta(x)=\min \{\varepsilon(x), d(x, \Phi \circ f(K))\}$. Let $\phi_{i}$ be the $i$-th component of the map $\Phi \circ f$. By local homotopy negligibility of $Q \backslash \bigcap_{\gamma \in \Gamma} X_{\gamma}$ there exists a homotopy $h:[0,1] \times Q \rightarrow Q$ with $h(0, x)=x, h((0,1] \times Q) \subset \bigcap_{\gamma \in \Gamma} X_{\gamma}$ and $h(1, x)=*$. Define a homotopy $H_{k}:[0,1] \times Q \rightarrow Q$ by

$$
H_{k}(t, x)= \begin{cases}h(2-2 t, x), & \text { if } \frac{1}{2} \leq t \leq 1, \\ h_{k}(2 t, x), & \text { if } 0 \leq t \leq \frac{1}{2},\end{cases}
$$

where $h_{k}:[0,1] \times Q \rightarrow Q$ is a homotopy such that $h_{k}((0,1] \times Q) \subset$ $\bigcap_{y \in \Gamma} X_{\gamma}, h_{k}(0, x)=\phi_{k}(x)$ and $h_{k}(1, x)=*$. For $x \in\{y \in Q$ : $\left.2^{-k-1} \leq \delta(\Phi \circ f(y)) \leq 2^{-k}\right\}, k=1,2, \ldots$, define

$$
\begin{array}{r}
f^{\prime}(x)=\left(\phi_{1}(x), \phi_{2}(x), \ldots, \phi_{k}(x), H_{k+1}\left(-k-\log _{2} \delta(\Phi \circ f(x)), x\right),\right. \\
\left.x, x, h\left(-k-\log _{2} \delta(\Phi \circ f(x)), x\right), *, *, \ldots\right)
\end{array}
$$

and extend $f^{\prime}$ on $K$ by $f^{\prime}|K=\Phi \circ f| K$. By the construction $f^{\prime}: Q \rightarrow Q^{\mathbf{N}}$ is a continuous, one-to-one map which is $\varepsilon^{\prime}$-close to $\Phi \circ f$. Moreover, $\left(f^{\prime}\right)^{-1}\left(X_{\gamma}^{\mathbf{N}}\right) \backslash K=X_{\gamma} \backslash K$ and $f^{\prime}\left(X_{\gamma} \backslash K\right) \subset W\left(X_{\gamma}, *\right)$. Hence, the map $g=\Phi^{-1} \circ f^{\prime}$ is a Z-embedding which is $\varepsilon$-close to $f$ and satisfies $g^{-1}\left(X_{\gamma}\right) \backslash K=X_{\gamma} \backslash K$.

Let $\Phi: \widehat{\mathbf{R}}^{\mathbf{N}} \rightarrow\left(\widehat{\mathbf{R}}^{\mathbf{N}}\right)^{\mathbf{N}}$ be any map that simply rearranges coordinates. It is easily seen that with this map the system $\Sigma$ satisfies the conditions of Lemma 6.2. So we have:

THEOREM 6.3. The system $\Sigma$ is $\mathscr{F}_{\sigma}$-absorbing and $c_{0}$ is an $\mathscr{F}_{\sigma \delta^{-}}$ absorber in $Q$.

The space $\mathbf{R}_{f}^{\mathbf{N}}$ is defined as $W(\mathbf{R}, 0)$. This space is homeomorphic to $l_{f}^{2}$ and furthermore the pair $\left(\widehat{\mathbf{R}}^{\mathbf{N}}, \mathbf{R}_{f}^{\mathbf{N}}\right)$ is homeomorphic to $\left(I^{\mathbf{N}}, \sigma\right)$. This means, according to $\S 3$ that there exists a homeomorphism $\alpha: Q \rightarrow Q^{\mathbf{N}}$ such that for every $k \in \mathbf{N}$,

$$
\alpha\left(\Sigma_{k}\right)=\underbrace{\mathbf{R}_{f}^{\mathbf{N}} \times \cdots \times \mathbf{R}_{f}^{\mathbf{N}}}_{k \text { times }} \times Q \times Q \times \cdots .
$$

Consequently, $c_{0}$ is mapped by $\alpha$ onto $\left(\mathbf{R}_{f}^{\mathbf{N}}\right)^{\mathbf{N}}$. In [9, Question 6.11] the following problem is posed. Does there exist a homeomorphism from $\mathbf{R}^{\mathbf{N}}$ onto $\left(\mathbf{R}^{\mathbf{N}}\right)^{\mathbf{N}}$ that maps $c_{0}$ onto $\left(\mathbf{R}_{f}^{\mathbf{N}}\right)^{\mathbf{N}}$ ? Such a homeomorphism cannot exist because $c_{0}$ is contained in the $\sigma$-compactum 
consisting of bounded sequences where as $\left(\mathbf{R}_{f}^{\mathbf{N}}\right)^{\mathbf{N}}$ contains a copy of $\mathbf{R}^{\mathbf{N}}$ that is closed in $\left(\mathbf{R}^{\mathbf{N}}\right)^{\mathbf{N}}$.

LEMMA 6.4. If $A$ is strongly $\mathscr{M}$-universal in $Q$ and $X$ is locally homotopy negligible in a compact absolute retract $M$ then $A \times(M \backslash X)$ is strongly $\mathscr{M}$-universal in $Q \times M$.

Proof. This is similar to the proof of Theorem 3.1. Let $f=\left(f_{1}, f_{2}\right)$ be a Z-embedding of $Q$ in $Q \times M$. Let $K$ and $C$ be subsets of $Q$ such that $K$ is closed and $C$ is an element of $\mathscr{M}$. Select a map $\varepsilon: Q \rightarrow I$ such that $\varepsilon^{-1}(0)=K$ and $\varepsilon(x) \leq \rho(f(x), f(K))$ for each $x \in Q$. Just as in the proof of Theorem 3.1 we can find a map $g_{1}: Q \rightarrow Q$ such that $f_{1}$ and $g_{1}$ are $\varepsilon$-close, $g_{1}^{-1}(A) \backslash K=C \backslash K$, $g_{1} \mid Q \backslash K$ is a one-to-one map whose range is a $\sigma$ Z-set. Since $X$ is locally homotopy negligible we can find a map $g_{2}: Q \rightarrow M$ such that $f_{2}$ and $g_{2}$ are $\varepsilon$-close and $g_{2}(Q \backslash K) \subset M \backslash X$. The map $g=$ $\left(g_{1}, g_{2}\right)$ is a Z-embedding of $Q$ into $Q \times M$ with $g|K=f| K$ and $g^{-1}(A \times(M \backslash X)) \backslash K=C \backslash K$.

THEOREM 6.5. If $X$ is a countable, nondiscrete metric space then $C_{p}(X)$ is an $\mathscr{F}_{\sigma \delta}$-absorber in $\widehat{\mathbf{R}}^{X}$.

This means that there exists a homeomorphism $\beta: \widehat{\mathbf{R}}^{X} \rightarrow Q^{\mathbf{N}}$ such that $\beta\left(C_{p}(X)\right)=\left(\mathbf{R}_{f}^{\mathbf{N}}\right)^{\mathbf{N}}$.

Proof. It is well known (and easily verified) that $C_{p}(X)$ is an element of $\mathscr{F}_{\sigma \delta}$. Let $A$ be a convergent sequence in $X$. Observe that $\bigcup_{n=1}^{\infty}\left\{f \in \widehat{\mathbf{R}}^{X}:|f(a)| \leq n\right.$ for every $\left.a \in A\right\}$ is a $\sigma$ Z-set that contains $C_{p}(X)$. It remains to be shown that $C_{p}(X)$ is strongly $\mathscr{F}_{\sigma \delta}$-universal.

We first prove this for the convergent sequence $\widehat{\mathbf{N}}=\mathbf{N} \cup\{\infty\}$. In $\widehat{\mathbf{R}}$ and $\widehat{\mathbf{N}}$ we use the following arithmetic: $1 / 0=\infty$ and $\infty+a=\infty$ if $a$ is finite. Define the following continuous function from $\widehat{\mathbf{R}}$ into $\widehat{\mathbf{R}}^{\widehat{N}}$ :

$$
\Psi(r)(n)=\operatorname{sign}(r) \min \{|r|, n\} .
$$

Note that $\Psi(r)(n)$ is finite if $n \neq \infty$ and $\lim _{n \rightarrow \infty} \Psi(r)(n)=\Psi(r)(\infty)$ $=r$. This means that $\Psi(\mathbf{R})$ is a subset of $C_{p}(\widehat{\mathbf{N}})$. If $f \in \widehat{\mathbf{R}}^{\mathbf{N}}$ then $\hat{f}$ is the extension of $f$ over $\widehat{\mathbf{N}}$ that assigns 0 to $\infty$. It is easily seen that $\boldsymbol{\Phi}(f, r)=\hat{f}+\Psi(r)$ is a well-defined map from $\widehat{\mathbf{R}}^{\mathbf{N}} \times \widehat{\mathbf{R}}$ onto $\widehat{\mathbf{R}}^{\widehat{\mathbf{N}}}$. Observing that $\Phi^{-1}(h)=(h-\Psi(h(\infty)) \mid \mathbf{N}, h(\infty))$ we find that $\Phi$ is a homeomorphism. Note that $\Phi\left(c_{0} \times \mathbf{R}\right)=C_{p}(\widehat{\mathbf{N}})$. According 
to Lemma $6.4 c_{0} \times \mathbf{R}$ is strongly $\mathscr{F}_{\sigma \delta}$-universal in $Q \times \widehat{\mathbf{R}}$ and hence $C_{p}(\widehat{\mathbf{N}})$ is strongly $\mathscr{F}_{\sigma \delta}$-universal in $\widehat{\mathbf{R}} \widehat{\mathbf{N}}$.

We use a similar argument to reduce the problem for $C_{p}(X)$ to $C_{p}(\widehat{\mathbf{N}})$. Let $d$ be a metric on $X$ and let $A$ be a convergent sequence in $X$. We may assume that $C_{p}(A)$ is strongly $\mathscr{F}_{\sigma \delta}$-universal in $\widehat{\mathbf{R}}^{A}$. Choose a retraction $r$ from $X$ onto $A$. The formula

$$
\Psi(g)(x)=\operatorname{sign}(g(r(x))) \min \{|g(r(x))|, 1 / d(x, r(x))\}
$$

defines a continuous selection that extends every $g \in \widehat{\mathbf{R}}^{A}$ to an element of $\widehat{\mathbf{R}}^{X}$. The map $\Psi$ has the following properties: $\Psi(g) \mid A=\dot{g}$, $\Psi(g) \mid X \backslash A$ has its values in $\mathbf{R}$ and $\Psi\left(C_{p}(A)\right) \subset C_{p}(X)$. If $f \in \widehat{\mathbf{R}}^{X \backslash A}$ then $\hat{f}$ is the extension of $f$ over $X$ with zeros. As above it is easily seen that $\Phi(f, g)=\hat{f}+\Psi(g)$ is a well-defined map from $\widehat{\mathbf{R}}^{X \backslash A} \times \widehat{\mathbf{R}}^{A}$ onto $\widehat{\mathbf{R}}^{X}$ and a homeomorphism. Let $C_{p}(X, A)$ stand for $\{f \mid X \backslash A$ : $f \in C_{p}(X)$ and $f \mid A=0$ \} and note that $\Phi\left(C_{p}(X, A) \times C_{p}(A)\right)=$ $C_{p}(X)$. It is easily seen that the complement of $C_{p}(X, A)$ in $\widehat{\mathbf{R}}^{X \backslash A}$ is locally homotopy negligible and hence Lemma 6.4 implies that $C_{p}(X)$ is strongly $\mathscr{F}_{\sigma \delta}$-universal in $\widehat{\mathbf{R}}^{X}$. This completes the proof of Theorem 6.5 .

\section{REFERENCES}

[1] R. D. Anderson, D. W. Curtis and J. van Mill, A fake topological Hilbert space, Trans. Amer. Math. Soc., 272 (1982), 311-321.

[2] M. Bestvina and J. Mogilski, Characterizing certain incomplete infinite-dimensional absolute retracts, Michigan Math. J., 33 (1986), 291-313.

[3] R. Cauty, L'espace des functions continues d'un espace métrique dénombrable, preprint.

[4] D. W. Curtis, Boundary sets in the Hilbert cube, Topology Appl., 20 (1985), 201-221.

[5] _ Hyperspaces of finite sets as boundary sets, Topology Appl., 22 (1986), 97-107.

[6] D. W. Curtis and R. M. Schori, Hyperspaces of Peano continua are Hilbert cubes, Fund. Math., 101 (1978), 19-38.

[7] J. J. Dijkstra, T. Dobrowolski, W. Marciszewski, J. van Mill and J. Mogilski, Recent classification and characterization results in geometric topology, Bull. Amer. Math. Soc. (N.S.), 22 (1990), 277-283.

[8] T. Dobrowolski, S. P. Gul'ko and J. Mogilski, Function spaces homeomorphic to the countable product of $l_{2}^{f}$, Topology Appl., 34 (1990), 153-160.

[9] T. Dobrowolski and J. Mogilski, Problems on topological classification of incomplete metric spaces, Open Problems in Topology (J. van Mill and G. M. Reed, eds.), North-Holland, Amsterdam, 1990.

[10] N. Kroonenberg, Pseudo-interiors of hyperspaces, Compositio Math., 32 (1976), 113-131. 
[11] J. van Mill, Topological equivalence of certain function spaces, Compositio Math., 63 (1987), 159-188.

[12] __ Infinite-Dimensional Topology, Prerequisites and Introduction, NorthHolland, Amsterdam, 1989.

[13] H. Toruńczyk, Concerning locally homotopy negligible sets and characterization of $l_{2}$-manifolds, Fund. Math., 101 (1978), 93-110.

Received April 15, 1990 and in revised form May 3, 1991.

The University of Alabama

Box 870350

Tuscaloosa, Alabama 35487-0350

(J. J. Dijkstra and J. Mogilski)

AND

VRIJE UNIVERSITEIT

Postbus 7161

1007 MC AMSterdam, The Netherlands

(J. van Mill)

Current address, J. Mogilski: Bradley University

Peoria, IL 61625 



\title{
PACIFIC JOURNAL OF MATHEMATICS EDITORS
}

V. S. VARADARAJAN

(Managing Editor)

University of California

Los Angeles, CA 90024-1555-05

HeRbert Clemens

University of Utah

Salt Lake City, UT 84112

F. Michael Christ

University of California

Los Angeles, CA 90024-1555

THOMAS ENRIGHT

University of California, San Diego

La Jolla, CA 92093
Nicholas ERcolani

University of Arizona

Tucson, AZ 85721

R. FINN

Stanford University

Stanford, CA 94305

VAUGHAN F. R. JONES

University of California

Berkeley, CA 94720

Steven Kerckhoff

Stanford University

Stanford, CA 94305

\section{C. MOORE}

University of California

Berkeley, CA 94720

Martin ScharlemanN

University of California

Santa Barbara, CA 93106

HAROLD STARK

University of California, San Diego

La Jolla, CA 92093

\section{ASSOCIATE EDITORS}
R. Arens
E. F. BECKENBACH
B. H. NeumanN
F. WoLF
K. YoshidA
(1906-1982)
(1904-1989)

\section{SUPPORTING INSTITUTIONS}

UNIVERSITY OF ARIZONA

UNIVERSITY OF BRITISH COLUMBIA

CALIFORNIA INSTITUTE OF TECHNOLOGY

UNIVERSITY OF CALIFORNIA

MONTANA STATE UNIVERSITY

UNIVERSITY OF NEVADA, RENO

NEW MEXICO STATE UNIVERSITY

OREGON STATE UNIVERSITY

\author{
UNIVERSITY OF OREGON \\ UNIVERSITY OF SOUTHERN CALIFORNIA \\ STANFORD UNIVERSITY \\ UNIVERSITY OF HAWAII \\ UNIVERSITY OF TOKYO \\ UNIVERSITY OF UTAH \\ WASHINGTON STATE UNIVERSITY \\ UNIVERSITY OF WASHINGTON
}

The Supporting Institutions listed above contribute to the cost of publication of this Journal, but they are not owners or publishers and have no responsibility for its content or policies.

\begin{abstract}
Mathematical papers intended for publication in the Pacific Journal of Mathematics should be in typed form or offset-reproduced (not dittoed), double spaced with large margins. Please do not use built up fractions in the text of the manuscript. However, you may use them in the displayed equations. Underline Greek letters in red, German in green, and script in blue. The first paragraph must be capable of being used separately as a synopsis of the entire paper. In particular it should contain no bibliographic references. Please propose a heading for the odd numbered pages of less than 35 characters. Manuscripts, in triplicate, may be sent to any one of the editors. Please classify according to the 1991 Mathematics Subject Classification scheme which can be found in the December index volumes of Mathematical Reviews. Supply name and address of author to whom proofs should be sent. All other communications should be addressed to the managing editor, or Elaine Barth, University of California, Los Angeles, California 90024-1555-05.

There are page-charges associated with articles appearing in the Pacific Journal of Mathematics. These charges are expected to be paid by the author's University, Government Agency or Company. If the author or authors do not have access to such Institutional support these charges are waived. Single authors will receive 50 free reprints; joint authors will receive a total of 100 free reprints. Additional copies may be obtained at cost in multiples of 50 .
\end{abstract}

The Pacific Journal of Mathematics (ISSN 0030-8730) is published monthly except for July and August. Regular subscription rate: $\$ 190.00$ a year (10 issues). Special rate: $\$ 95.00$ a year to individual members of supporting institutions.

Subscriptions, orders for numbers issued in the last three calendar years, and changes of address should be sent to Pacific Journal of Mathematics, P.O. Box 969, Carmel Valley, CA 93924, U.S.A. Old back numbers obtainable from Kraus Periodicals Co., Route 100, Millwood, NY 10546.

The Pacific Journal of Mathematics at P.O. Box 969, Carmel Valley, CA 93924 (ISSN 0030-8730) is published monthly except for July and August. Second-class postage paid at Carmel Valley, California 93924, and additional mailing offices. Postmaster: send address changes to Pacific Journal of Mathematics, P.O. Box 969, Carmel Valley, CA 93924.

PUBLISHED BY PACIFIC JOURNAL OF MATHEMATICS, A NON-PROFIT CORPORATION Copyright (C) 1992 by Pacific Journal of Mathematics 


\section{Pacific Journal of Mathematics}

Vol. 152, No. $2 \quad$ February, 1992

Edoardo Ballico, On the restrictions of the tangent bundle of the

Grassmannians

Edward Burger, Homogeneous Diophantine approximation in

$S$-integers

Jan Dijkstra, Jan van Mill and Jerzy Mogilski, The space of

infinite-dimensional compacta and other topological copies of $\left(l_{f}^{2}\right)^{\omega} \ldots 255$

Mike Hoffman, Multiple harmonic series ..................... 275

Wu Hsiung Huang, Superharmonicity of curvatures for surfaces of constant

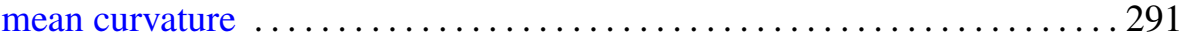

George Kempf, Pulling back bundles ......................... 319

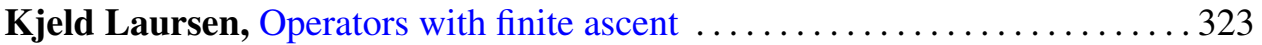

Andrew Solomon Lipson, Some more states models for link invariants . . . . 337

Xiang Yang Liu, Bloch functions of several complex variables .......... 347

Madabusi Santanam Raghunathan, A note on generators for arithmetic

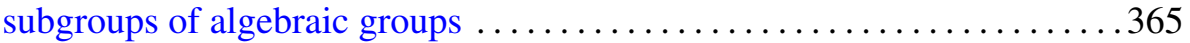

Marko Tadić, Notes on representations of non-Archimedean $\operatorname{SL}(n) \ldots \ldots 375$ 